\section{Nodular lesions of the liver in multiple myeloma}

Haematologica 2007; 92:(7)e81

An 83-year-old woman was admitted because of asthenia, weight loss and abdominal pain. She had been diagnosed with MGUS IgGk twenty years earlier and the monoclonal protein levels had remained persistently stable. At admission, physical examination showed pallor, marked hepatomegaly ( $5 \mathrm{~cm}$ below the costal margin) and a gross epigastric mass. A blood count revealed moderate macrocytic anemia. Liver function tests showed a low level of pseudocholinesterase and increased gamma-glutamyl-transpeptidase. Kidney biochemical tests and serum calcium were normal. Serum protein electrophoresis showed a remarkable increase of the $\mathrm{M}$-component (9.2 g/dL, IgGk). Bone marrow was hypocellular with moderate infiltration by atypical plasma cells. Total skeletal X-rays showed diffuse osteoporosis. Abdominal ultrasonography (US) revealed several nodular hepatic lesions of various sizes in both lobes (Figure 1A), the largest one $(6 \mathrm{~cm})$ in the left lobe (Figure 1B). Most of the lesions were hypoechogenic with a hyperechogenic halo suggesting metastatic disease. A US-guided fine-needle aspiration of one of the hepatic lesions was performed. Numerous large immature plasma cells, showing central or eccentric nucleus with loose chromatin, prominent nucleoli and deeply basophilic cytoplasm were observed (Figures 2AD). Cytoplasmic kappa light chains were shown by immunocytochemistry (Figure 2D, insert). There were also a few scattered or clustered normal hepatocytes (Figures 2A and C). Liver histology confirmed the infiltration by $\mathrm{CD}_{138^{+}}, \mathrm{CD} 20^{+}$, CD59- and EMA- plasma cells. Thus, a final diagnosis of multiple myeloma with liver involvement was made. The patient was treated with dexamethasone and bortezomib with a good clinical improvement.

Diffuse hepatic infiltration occurs in more than $50 \%$ of cases with multiple myeloma, especially at the end stage. ${ }^{1}$ Liver involvement by multiple myeloma as space-occupying lesions is a very rare condition, that mimics metastatic tumours on imaging techniques. ${ }^{2-6}$ Immunocytochemistry for cytoplasmic Ig light chains is helpful for the differential diagnosis.

Rosangela Invernizzi, Gabriella Carnevale Maffe, Erica Travaglino, Elisabetta Pagani, Carla Pieresca Department of Internal Medicine, University of Pavia and IRCCS Policlinico S. Matteo Foundation, Pavia, Italy

Correspondence: R. Invernizzi, M.D.

Clinica Medica III, Policlinico S. Matteo, Piazzale Golgi, 27100

Pavia, Italy

E-mail:r.invernizzi@smatteo.pv.it
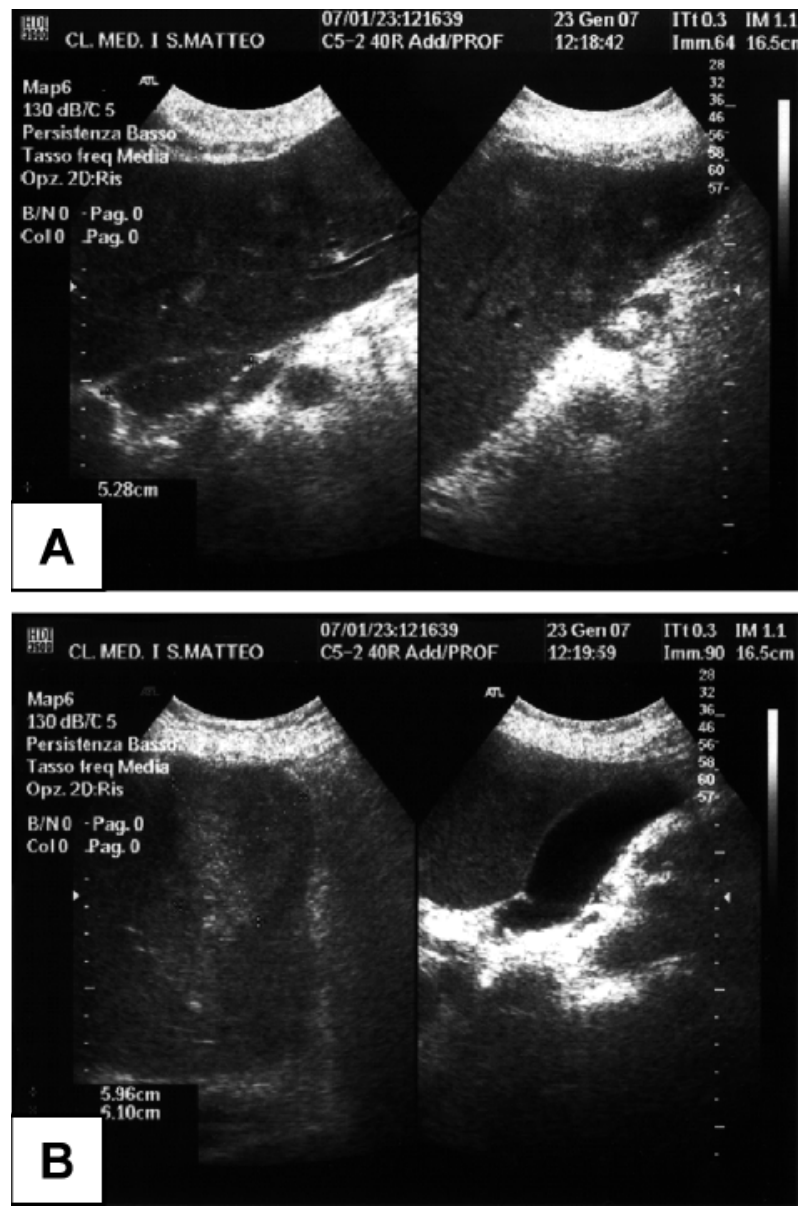

Figure 1.

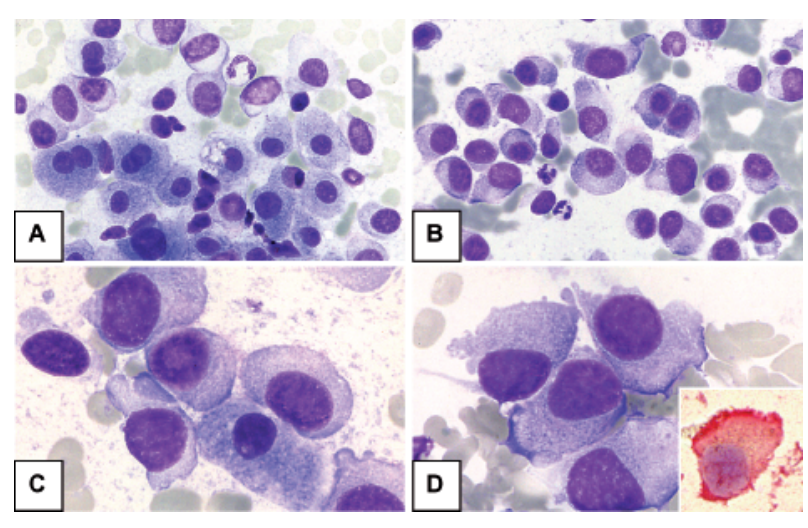

Figure 2.

Launay $\bigcirc$, et al. Nodular lesions of the liver revealing multiple myeloma. Leuk Lymphoma 1999 ; 33 :389-92.

4. Monill J, Pernas J, Montserrat E, Perez C, Clavero J, MartinezNoguera A, et al. CT features of abdominal plasma cell neoplasms. Eur Radiol 2005; 15:1705-12.

5. Galani Z, Viniou N, Kyrtsonis MC, Sachanas S, Antoniou T, Dimopoulou MN, et al. Hepatic and renal plasma cell lesions in a patient with multiple myeloma in hematological remission. Anticancer Research 2007; 27:571-4. 